\title{
AN UPPER BOUND FOR THE CURVATURE INTEGRAL
}

\author{
A. M. PETRUNIN
}

\begin{abstract}
It is shown that the integral of the scalar curvature of a closed Riemannian manifold can be bounded from above in terms of the manifold's dimension,
\end{abstract} diameter, and a lower bound for the sectional curvature.

\section{§1. INTRODUCTION}

Our main result is as follows.

1.1. Theorem. Let $M$ be a complete Riemannian m-manifold with sectional curvature at least -1 . Then

$$
\int_{B_{1}(p)} \mathrm{Sc} \leq \operatorname{const}(m)
$$

for any $p \in M$, where $B_{1}(p)$ denotes the unit ball centered at $p \in M$ and $\mathrm{Sc}$ is the scalar curvature of $M$.

The following example shows that this result is optimal in a sense. Consider a convex polyhedron $P$ in $\mathbb{R}^{m+1}$, and let $\partial P_{\varepsilon}$ denote the surface of its $\varepsilon$-neighborhood (if necessary, one can smooth $\partial P_{\varepsilon}$ over to make it $C^{\infty}$-smooth). Then the $\partial P_{\varepsilon}$ are Riemannian $m$ manifolds with nonnegative sectional curvature, and the integral $\int_{\partial P_{\varepsilon}}$ Sc remains nearly constant for small $\varepsilon$, while for any $\theta>0$ we have

$$
\int_{\partial P_{\varepsilon}}|\mathrm{Sc}|^{1+\theta} \rightarrow \infty \text { as } \varepsilon \rightarrow 0 .
$$

Note that if $P$ is of codimension 2 or 3 , then this construction gives an example of families of collapsing Riemannian manifolds $\partial P_{\varepsilon}$ with the integral $\int_{\partial P_{\varepsilon}}$ Sc bounded from below by a positive constant. Corollary 1.3 shows that this is no longer possible if the dimension drops by 3 or more under the collapse.

1.2. Variations and generalizations. The Bishop-Gromov inequality implies that any ball of radius $R$ in a complete Riemannian $m$-manifold with sectional curvature at least -1 can be covered by $\exp (4 m R)$ unit balls. This implies that, under the assumptions of the theorem, we have the estimate:

$$
\int_{B_{R}(p)} \mathrm{Sc} \leq \operatorname{const}(m) \exp (4 m R) .
$$

On the other hand, rescaling shows that, for $R<1$,

$$
\int_{B_{R}(p)} \mathrm{Sc} \leq \operatorname{const}(m) R^{m-2} .
$$

Together, these two inequalities yield the following statement.

2000 Mathematics Subject Classification. Primary 53B21.

Key words and phrases. Sectional curvature, scalar curvature, Aleksandrov space. 
1.3. Corollary. Let $M$ be a complete Riemannian m-manifold with sectional curvature at least -1 , and let $p \in M$. Then

$$
\int_{B_{R}(p)} \operatorname{Sc} \leq \operatorname{const}(m) R^{m-2} \exp (4 m R) .
$$

It is easily seen that if all sectional curvatures are at least -1 , then for the Riemann curvature tensor Rm we have

$$
|\mathrm{Rm}| \leq \mathrm{Sc}+m^{2} .
$$

Therefore, the above corollary also implies that

$$
\int_{B_{R}(p)}|\mathrm{Rm}| \leq \operatorname{const}^{\prime}(m) R^{m-2} \exp (4 m R),
$$

where

$$
\operatorname{const}^{\prime}(m)=\operatorname{const}(m)+m^{2} .
$$

1.4. I believe that in 1996 Gromov asked me whether the integral of the scalar curvature could be controlled in terms of the diameter and a lower curvature bound. However, after 10 years I cannot be sure that it was exactly the question he asked.

My proof is similar to Perelman's proof of the continuity of the integral of the scalar curvature

$$
\mathcal{F}(M)=\int_{M} \mathrm{Sc}
$$

on the set of all Riemannian $m$-manifolds with a uniform lower curvature bound. Perelman's proof involves exhausting the manifold by convex hypersurfaces (that proof is included as an appendix to [P-2003]). In fact, a slight modification of Perelman's proof makes it possible to prove the main theorem in the case where no collapse occures, i.e., under the following additional assumption:

$$
\operatorname{vol}\left(B_{1}(p)\right) \geq v_{0}>0 .
$$

I invoke a special form of Bochner's formula (see Subsection 2.2); a similar idea was used by Sergei Buyalo for obtaining lower estimates of the integral of scalar curvature on a 3-manifold Buy. This formula makes it possible to estimate the curvature not only in tangent but also in normal directions to the exhausting surfaces (I need this to make the proof work in the case of a collapse). Also, I need to use exhaustion by nested sequences of semiconvex surfaces, which causes additional technical difficulties.

The remaining part of the proof is a combination of the converging-rescaling technique.

1.5. Outline of the proof. Arguing by contradiction, assume that there is a sequence of $m$-manifolds $\left(M_{n}, p_{n}\right)$ with $K \geq-1$ such that

$$
\int_{B_{1}\left(p_{n}\right)} \mathrm{Sc} \rightarrow \infty .
$$

Applying Gromov's compactness theorem, we may assume that the $\left(M_{n}, p_{n}\right)$ converge to an Aleksandrov space $(A, p)$. Using the inductive hypothesis (the fact that a slightly more general statement is true for all smaller dimensions), we prove that the scalar curvature on $M_{n}$ converges weakly to a measure on $A$ that is finite everywhere except for a finite number of points. We choose one of these points $s \in A$, and blow $M_{n}$ up with carefully chosen marked points $s_{n} \in M_{n}$ near $s$, to make the curvature distribution visible, and then pass to the new limit space $\left(A^{\prime}, p^{\prime}\right)$. For $\left(A^{\prime}, p^{\prime}\right)$ we repeat the same procedure as for $(A, p)$, and so on. The statement follows, because only a finite number of such repetitions may occur, which is implied by the fact that, in a certain sense, $\left(A^{\prime}, p^{\prime}\right)$ is by a certain amount larger than $(A, p)$, and it cannot exceed $\left(\mathbb{R}^{m}, 0\right)$. 


\section{§2. The Bochner Formula}

Here we provide a version of the Bochner formula that relates the integral of the scalar curvature of a family of hypersurfaces to the integral of the Ricci curvature in their normal directions.

2.1. Notation. Let $M^{m+1}$ be a Riemannian $(m+1)$-manifold. Assume that $f: M^{m+1} \rightarrow$ $\mathbb{R}$ is a smooth function without critical values on $[a, b] \subset \mathbb{R}$ and that its level sets $L_{t}=f^{-1}(t)$ are compact for any $t \in[a, b]$. We denote:

(i) by $u=\nabla f /|\nabla f|$ the unit vector field normal to $L_{t}$;

(ii) by $\left\{e_{0}, e_{1}, e_{2}, \ldots, e_{m}\right\}$ an orthonormal frame such that $e_{0}=u$ and each $e_{i}, i>0$, goes in a principal direction of $L_{f(x)}$ at $x$ so that the corresponding principal curvatures

$$
\kappa_{i}=\left\langle\nabla_{e_{i}} u, e_{i}\right\rangle
$$

form a monotone nondecreasing sequence

$$
\kappa_{1}(x) \leq \kappa_{2}(x) \leq \cdots \leq \kappa_{m}(x)
$$

(iii) by $H(x)=\kappa_{1}(x)+\kappa_{2}(x)+\cdots+\kappa_{m}(x)$ the mean curvature of $L_{f(x)}$ at $x \in L_{f(x)}$;

(iv) by $G(x)=2 \sum_{i<j} \kappa_{i}(x) \kappa_{j}(x)$ the external term in the Gauss formula for the scalar curvature of $L_{t}$, i.e.,

$$
\mathrm{Sc}_{L}=2 \sum_{i<j}\left\langle R_{M}\left(e_{i}, e_{j}\right) e_{j}, e_{i}\right\rangle+G=\operatorname{Sc}_{M}-2 \operatorname{Ric}_{M}(u, u)+G .
$$

2.2. Bochner formula. In this notation, the Bochner formula can be written in the following form:

$$
\int_{f^{-1}([a, b])} \operatorname{Ric}_{M}(u, u)=\int_{f^{-1}([a, b])} G+\int_{L_{a}} H-\int_{L_{b}} H .
$$

Proof. We write the relative Bochner formula for the vector field $u=\nabla f /|\nabla f|$ in the domain $f^{-1}([a, b])$ :

$$
\int_{f^{-1}([a, b])}\langle D u, D u\rangle-\langle\nabla u, \nabla u\rangle=\int_{f^{-1}([a, b])} \operatorname{Ric}_{M}(u, u)-\int_{L_{a}} H+\int_{L_{b}} H .
$$

Since $e_{0}=u$ and $\left\langle\nabla_{u} u, u\right\rangle=0$, we have

$$
D u=\sum_{i=0}^{m} e_{i} \cdot \nabla_{e_{i}} u=\sum_{i=1}^{m} \kappa_{i} e_{i} \cdot e_{i}+u \cdot \nabla_{u} u=\sum_{i=1}^{m} \kappa_{i}+u \wedge \nabla_{u} u,
$$

where "." denotes the Clifford multiplication. Again recalling that $\left\langle\nabla_{u} u, u\right\rangle=0$, we obtain

On the other hand,

$$
\langle D u, D u\rangle=\left(\sum_{i=1}^{m} \kappa_{i}\right)^{2}+\left|\nabla_{u} u\right|^{2} .
$$

whence

$$
\nabla u=\sum_{i=1}^{m} \kappa_{i} e_{i} \otimes e_{i}+\nabla_{u} u \otimes u,
$$

Therefore,

$$
\langle\nabla u, \nabla u\rangle=\sum_{i=1}^{m} \kappa_{i}^{2}+\left|\nabla_{u} u\right|^{2} .
$$

This implies $(*)$.

$$
\langle D u, D u\rangle-\langle\nabla u, \nabla u\rangle=2 \sum_{i<j} \kappa_{i} \kappa_{j}=G
$$




\section{§3. Constructions in Aleksandrov geometry}

This section contains all the necessary technical results in Aleksandrov geometry, mainly related to corner surfaces. A corner surface is a generalization of semiconvex hypersurfaces to all codimensions. Roughly speaking, it is an intersection of semiconvex hypersurfaces that have acute angles with one another.

These level sets will be used in the proof in the same way as convex hypersurfaces in Perelman's original proof.

Everywhere in this section we use the notation and conventions of $\mathrm{P}-2007$.

The following definition is very similar to the definition of the strained (burst) points in $\mathrm{BGP}$, but I use it for submanifolds and add an additional parameter $\ell$ that describes how wide the strainers should be.

3.1. Definition. A subset $M$ of an Aleksandrov space $N$ is a $(k, \delta, \ell)$-corner surface if there is a collection of $1 / \ell$-concave functions $f_{i}, g_{i}: N \rightarrow \mathbb{R}, i \in\{1, \ldots, k\}$ (called the strainers of $M)$, defined in the $\ell$-neighborhood of $M$ and such that

$$
M=\left\{x \in N \mid f_{i}(x)=0 \text { for all } 1 \leq i \leq k\right\},
$$

and the collection $f_{i}, g_{i}: N \rightarrow \mathbb{R}$ is $\delta$-strained, which means that

(i) all $f_{i}, g_{i}$ are 1-Lipschitz;

(ii) for all $i \neq j$ we have

$$
\left|d f_{i}\left(\nabla g_{j}\right)\right|,\left|d g_{i}\left(\nabla f_{j}\right)\right|,\left|d g_{i}\left(\nabla g_{j}\right)\right|,\left|d f_{i}\left(\nabla f_{j}\right)\right| \leq \delta
$$

(iii) $d f_{i}\left(\nabla g_{i}\right), d g_{i}\left(\nabla f_{i}\right) \leq-1+2 \delta$.

Finally, the collection $f_{i}: N \rightarrow \mathbb{R}$ is tight, i.e.,

(iv) for any $x \in M$ and all $i \neq j$ we have

$$
d f_{i}\left(\nabla f_{j}\right) \leq 0 .
$$

Moreover, if $N$ is a smooth Riemannian manifold and all functions $\left\{f_{i}\right\}$ are smooth, then $M$ is called a smooth $(k, \delta, \ell)$-corner surface.

Remarks. In this definition one can take $k=0$, and in this case $M=N$.

Conditions (i)-(iv) guarantee that the functions $f_{i}$ have no critical points, and their level sets intersect at acute angles close to $\pi / 2$.

Note that if we rescale the metric on $N$ with factor $\lambda$, then a $(k, \delta, \ell)$-corner surface $M$ becomes a $(k, \delta, \lambda \ell)$-corner surface in $\lambda N$ with strainers $\left\{\lambda f_{i}, \lambda g_{i}\right\}$.

3.2. Limits of strained level sets. Suppose we have a sequence of $q$-manifolds $\left(N_{n}, p_{n}\right)$ with marked points and with sectional curvature at least -1 . Let $M_{n} \subset N_{n}$ be a sequence of $(k, \delta, \ell)$-corner surfaces determined by collections of strainers

$$
\left\{f_{i, n}, g_{i, n}\right\}, \quad i \in\{1, \ldots, k\}, \quad f_{i, n}, g_{i, n}: N_{n} \rightarrow \mathbb{R} .
$$

Passing to a subsequence of $N_{n}$, we may assume that

(i) $\left(N_{n}, p_{n}\right) \stackrel{\mathrm{GH}}{\longrightarrow}(N, p)$;

(ii) $M_{n} \rightarrow M \subset N$;

(iii) for each $i \in\{1, \ldots, k\}, f_{i, n} \rightarrow f_{i}: N \rightarrow \mathbb{R}$ and $g_{i, n} \rightarrow g_{i}: N \rightarrow \mathbb{R}$.

Clearly $N$ is an Aleksandrov space of dimension at most $q$ with curvature at least -1 , and $M$ is a $(k, \delta, \ell)$-corner surface with strainers $f_{i}, g_{i}: N \rightarrow \mathbb{R}$.

Moreover, if $M$ is compact and $m=q-k$, then

$$
\operatorname{vol}_{m} M=\lim _{n \rightarrow \infty} \operatorname{vol}_{m} M_{n} .
$$

This statement follows from the proof of [BGP, Theorem 10.8]. 
3.3. A dimension-like invariant. Here we introduce an invariant of Aleksandrov spaces, which has properties similar to those of dimension, but is slightly more sensitive.

Let $A$ be an Aleksandrov space with dimension not exceeding $q$. Given a positive number $\theta>0$, we define $\operatorname{dir}_{\theta} A$ to be

$$
\operatorname{dir}_{\theta} A=\min _{x \in A} \operatorname{pack}_{\theta} \Sigma_{x} A
$$

where $\Sigma_{x} A$ is the space of directions at $x \in A$, and $\operatorname{pack}_{\theta} \Sigma_{x} A$ denotes the maximum number of points in $\Sigma_{x} A$ lying at a distance exceeding $\theta$ from one another.

Clearly, $\operatorname{dir}_{\theta} A$ is an integer and

$$
\operatorname{dir}_{\theta} A \leq \operatorname{const}(\theta, \operatorname{dim} A) \leq \operatorname{pack}_{\theta} S^{q} .
$$

3.4. A lifting procedure. Here we introduce a special way to lift a point from an Aleksandrov space to a nearby Riemannian manifold. More precisely, we describe a way to lift a spire (see below) on a $(k, \delta, \ell)$-corner surface in an Aleksandrov space to a nearby $(k, \delta, \ell)$-corner surface in a nearby Riemannian manifold.

This technique will be used only once, at the very end of the proof of the implication $\mathrm{B}_{m} \Rightarrow \mathrm{A}_{m}^{\prime}$ (see Subsection 4.6] in the proof of the Monster-Lemma 4.3); for the rest, any kind of lifting will do the job.

Assume that $N_{n} \rightarrow N$ is a sequence of Riemannian $q$-manifolds with curvature at least -1 converging to an Aleksandrov space $N$. Let $\delta>0$ be sufficiently small. Let $M_{n} \subset N_{n}$ be a sequence of $(k, \delta, \ell)$-corner surfaces converging to $M \subset N$ (see Subsection 3.2).

Consider a positive function $b$ on $M$ with the maximal possible value $b(x)$ such that

$$
\left|\nabla_{y} \operatorname{dist}_{x}\right|>1-\delta \text { for any } y \in B_{2 b(x)}(x) \backslash\{x\} \text { and } 2 b(x) \leq \max \{1, \delta \ell\} \text {. }
$$

3.5. Definition. A point $x$ on a $(k, \delta, \ell)$-corner surface is called a spire if for every $y \in M, y \neq x$, we have $b(y) \leq|x y|$.

We shall show that if $x$ is a spire, then there is a sequence of points $x_{n} \in M_{n}$ that converges to $x \in M$ and has the following property.

3.6. Property. Let $a_{n}$ be the minimum number such that

$$
\left|\nabla_{y} \operatorname{dist}_{x_{n}}\right|>1-\delta \quad \text { for all points } y \in N_{n} \text { with } a_{n}<\left|x_{n} y\right| \leq b .
$$

Then either $a_{n}=0$ for arbitrarily large $n$, or, if $\left(N^{\prime}, x^{\prime}\right)$ is a partial limit of $\left(\frac{1}{a_{n}} N_{n}, x_{n}\right)$, we have

$$
\operatorname{dir}_{\delta} N^{\prime}>\operatorname{dir}_{\delta} N \quad \text { (see Subsection } 3.3 \text { ). }
$$

3.7. Construction. For any $\tilde{x}_{n} \in N_{n}$, we define $a\left(\tilde{x}_{n}\right)=a_{\delta, b}\left(\tilde{x}_{n}\right)$ to be the minimum number such that

$$
\left|\nabla_{y} \operatorname{dist}_{\tilde{x}_{n}}\right|>1-\delta \quad \text { if } \quad a\left(\tilde{x}_{n}\right)<\left|\tilde{x}_{n} y\right| \leq b .
$$

Note that for any sequences $M_{n} \ni \bar{x}_{n} \rightarrow x$ and $M_{n} \ni y_{n} \rightarrow y \neq x$ we have

$$
\liminf _{n \rightarrow \infty}\left|\nabla_{y_{n}} \operatorname{dist}_{\bar{x}_{n}}\right| \geq\left|\nabla_{y} \operatorname{dist}_{x}\right|
$$

so that $a\left(\bar{x}_{n}\right) \rightarrow 0$ as $n \rightarrow \infty$.

Now, we fix one such sequence $\bar{x}_{n} \rightarrow x$ and take $x_{n} \in M_{n} \cap B_{r}\left(\bar{x}_{n}\right) \subset N_{n}$ with minimal possible $a\left(x_{n}\right)$; here $r$ is a sufficiently small fixed number.

Set $a_{n}=a\left(x_{n}\right)$; since $x$ is a spire, we have $x_{n} \rightarrow x$, and in particular, $\left(N_{n}, x_{n}\right) \rightarrow$ $(N, x)$.

It remains only to prove that the chosen sequence possesses Property 3.6 .

Assume that $a_{n}>0$ for all large $n$. We pass to a subsequence such that $\left(\frac{1}{a_{n}} N_{n}, x_{n}\right) \rightarrow$ $\left(N^{\prime}, x^{\prime}\right)$. Clearly, for any $\theta>0$ we have

$$
\operatorname{dir}_{\theta} N^{\prime} \geq \operatorname{pack}_{\theta} \Sigma_{x} N \geq \operatorname{dir}_{\theta} N .
$$


Therefore, it suffices to show that if $\theta=\delta$, then the first inequality is strict.

Suppose that equality occurs. Then there is a point $p \in N^{\prime} \operatorname{such}$ that $\operatorname{pack}_{\delta} \Sigma_{p} N^{\prime}=$ $\operatorname{pack}_{\delta} \Sigma_{x} N=s$. We show that if $p_{n} \in \frac{1}{a_{n}} N_{n}$ is a sequence of points converging to $p$, then $\frac{1}{a_{n}} a\left(p_{n}\right) \rightarrow 0$. In particular, for large $n$ we have $a\left(p_{n}\right)<a\left(x_{n}\right)$, which contradicts the choice of $x_{n}$ (here we denote by $p_{n}$ a point in $\frac{1}{a_{n}} N_{n}$ and also the respective point in $\left.N_{n}\right)$.

Choosing points $q_{1}, q_{2}, \ldots, q_{s} \in N$ such that $\angle q_{i} x q_{j}>\delta$ if $i \neq j$, we let $q_{i, n} \in N_{n}$ be a sequence converging to $q_{i} \in N$. Let $y_{n} \in N$ be a point such that $\left|p_{n} y_{n}\right|_{N} \rightarrow 0$ and $\mid \nabla_{y_{n}}$ dist $_{p_{n}} \mid \leq 1-\delta$. Then it is easy to show that $\angle y_{n} p_{n} q_{n} \geq 2 \delta$ for large $n$; passing to the limit as $n \rightarrow \infty$ we see that $\operatorname{pack}_{\delta} \Sigma_{x} N>s$, a contradiction.

\section{§4. Proof of Theorem 1.1}

4.1. Notation. Let $X$ be a Riemannian manifold, let $x \in X$, and let $\sigma$ be a sectional direction at $x$. We denote by $K_{X}(\sigma)$ the sectional curvature of $X$ in the direction $\sigma$ and set

$$
K_{X}^{ \pm}(x)=\max \left\{0, \max _{\sigma}\{ \pm K(\sigma)\}\right\}
$$

where $\sigma$ runs over all sectional directions at $x$.

Theorem 1.1 is a consequence of the following statement for $k=0$.

4.2. Theorem. There is $\delta=\delta(q, k)>0$ such that if $N$ is a Riemannian q-manifold with sectional curvature at least -1 and $M \subset N$ is a complete smooth $(k, \delta, \ell)$-corner surface, then

for any $x \in N$.

$$
\int_{M \cap B_{1}(x)} \operatorname{Sc}_{M}^{+} \leq \operatorname{const}(q, k, \ell)\left[1+\int_{M \cap B_{2}(x)} K_{M}^{-}\right]
$$

If $\operatorname{dim} M \geq 3$, this follows from statement $\mathrm{A}_{k}^{\prime}$ of the Monster-Lemma 4.3, and the case of $\operatorname{dim} M=2$ follows from the 3-dimensional case for $M \times S^{1} \subset N \times S^{1}$.

4.3. Monster-Lemma. There are constants $\mathcal{A}_{k}=\mathcal{A}(q, k, \ell), \mathcal{A}_{k}^{\prime}=\mathcal{A}^{\prime}(q, k, \ell)$, and $\mathcal{B}_{k}=\mathcal{B}(q, k, \ell)$ and a sequence of small positive constants $\delta_{k}, k=\{0,1, \ldots, q-3\}$, such that if

$N$ is a Riemannian q-manifold with sectional curvature at least -1, and

$M \subset N$ is a complete smooth $\left(k, \delta_{k}, \ell\right)$-corner surface,

then for any $k \in\{0,1, \ldots, q-3\}$ the following statements are true.

$\mathrm{A}_{k}$. If $\operatorname{diam} M \leq 1$, then

$$
\int_{M} \mathrm{Sc}_{M}^{+} \leq \mathcal{A}_{k}\left[1+\int_{M} K_{M}^{-}\right] .
$$

$\mathrm{A}_{k}^{\prime}$. For any $x \in N$ we have

$$
\int_{M \cap B_{1}(x)} \operatorname{Sc}_{M}^{+} \leq \mathcal{A}_{k}^{\prime}\left[1+\int_{M \cap B_{2}(x)} K_{M}^{-}\right] .
$$

$\mathrm{B}_{k}$. Assume that for some $x \in M$ we have $\left|\nabla_{y} \operatorname{dist}_{x}\right|>1-\delta_{k}$ for all $y \in N$ with $a<|x y|<2 b<\max \{1, \delta \ell\} ;$ then

$$
\int_{\operatorname{dist}_{x}^{-1}([2 a, b]) \cap M} \mathrm{Sc}_{M}^{+} \leq \mathcal{B}_{k}\left[1+\int_{\operatorname{dist}_{x}^{-1}([a, 2 b]) \cap M} K_{M}^{-}\right] .
$$


Proof. Clearly, $\mathrm{A}_{k}^{\prime} \Rightarrow \mathrm{A}_{k}$. Therefore, in order to prove the lemma, it suffices to prove statements $\mathrm{A}_{q-2}$ and the implications $\mathrm{A}_{k} \Rightarrow \mathrm{B}_{k-1}, \mathrm{~B}_{k} \Rightarrow \mathrm{A}_{k}^{\prime}$ for each $k$.

4.4. $\mathrm{A}_{q-2}$. In this case we have $\operatorname{dim} M=2$, so that $\mathrm{Sc}_{M}^{ \pm}=2 K_{M}^{ \pm}$. Now, the statement follows from the Gauss-Bonnet formula:

$$
\int_{M} K_{M}^{+}=\int_{M} K_{M}+\int_{M} K_{M}^{-} \leq 4 \pi\left(1+\int_{M} K_{M}^{-}\right) .
$$

Thus, we can take $\mathcal{A}_{q-2}=8 \pi$.

4.5. $\mathrm{A}_{k} \Rightarrow \mathrm{B}_{k-1}$. Let $\left\{f_{i}, g_{i}\right\}, i=\{1, \ldots, k-1\}$, be a set of strainers for $M$. We consider the function $f: M \rightarrow \mathbb{R}$ defined by the formula

$$
f(y)=\left(1-\delta_{k}\right) \widetilde{\operatorname{dist}}_{x}(y)+\frac{\delta_{k}}{k} \sum_{i=1}^{k-1}\left(g_{i}(y)-g_{i}(x)\right),
$$

where $\widetilde{\text { dist }_{x}}$ denotes a smoothing of the distance function dist ${ }_{x}$. Clearly, on the set $\operatorname{dist}^{-1}([a, 2 b]) \cap M$, we have

$$
1-2 \delta_{k} \leq \frac{f(y)}{\operatorname{dist}_{x} y}<1
$$

Therefore, to prove $\mathrm{B}_{k-1}$, it suffices to show that for some constant $\mathcal{B}(q, k, \ell)$ we have

$$
\int_{f^{-1}([a, b])} \operatorname{Sc}_{M}^{+} \leq \mathcal{B}(q, k, \ell)\left[1+\int_{f^{-1}\left(\left[a, \frac{3}{2} b\right]\right)} K_{M}^{-}\right] .
$$

On the set $\operatorname{dist}^{-1}([a, 2 b]) \cap M$, the function $f$ behaves like dist $x$, and it is smooth and its level subsets form corner surfaces in $N$ (so we can apply $\mathrm{A}_{k}$ ). Moreover, the following is true (compare with [BGP, 11.8]).

The function $f$ is semiconcave in a neighborhood of $f^{-1}\left(\left[a, \frac{3}{2} b\right]\right)$, and for some $\alpha=$ $\alpha(q, k, \ell)>0$ we have:

(i) $1 \geq|\nabla f|>1 / \alpha$ everywhere in $f^{-1}\left(\left[a, \frac{3}{2} b\right]\right)$ on $M$, and in particular, $f: M \rightarrow \mathbb{R}$ has no critical values on $\left[a, \frac{3}{2} b\right]$;

(ii) for any $t \in\left[a, \frac{3}{2} b\right]$, the level set $L_{t}=f^{-1}(t)$ forms a compact smooth $\left(k, \delta_{k}, t / \alpha\right)$ strained level set in $N$, and if $m=\operatorname{dim} L_{t}=q-k$, then

a) $A(t) \stackrel{\text { def }}{=} \operatorname{vol}_{m} L_{t} \leq \alpha t^{m}$;

b) $\operatorname{diam} L_{t} \leq \alpha t$;

c) the principal curvatures of $L_{t}$ in $M$ are at most $\alpha / t$.

Namely, if $u=\nabla f /|\nabla f| \in T M$, then for any unit vector $v$ tangent to $L_{t}$ we have

$$
\left\langle\nabla_{v} u, v\right\rangle \leq \alpha / t
$$

where $\nabla$ denotes the Levi-Civita connection on $M$.

To prove that $L_{t}=f^{-1}(t)$ is a $\left(k, \delta_{k}, t / \alpha\right)$-corner surface in $N$, it suffices to add two functions to the collection of strainers of $M$, taking $f_{k}=f$ and $g_{k}=\operatorname{dist}_{L_{(1+\varepsilon) t}}$ for a sufficiently small constant $\varepsilon>0$. To prove the volume estimate, one can argue by contradiction, using the convergence of the volume of corner surfaces; see Subsection 3.2 . The details of the proof are left to the reader.

\section{Notation.}

(i) $\kappa_{1}(x) \leq \kappa_{2}(x) \leq \cdots \leq \kappa_{m}(x)$ are the principal curvatures of $L_{t} \subset M$ at $x \in L_{t}$ with respect to $u$. 
(ii) $\beta:[a, b] \rightarrow \mathbb{R}_{+}$is an upper bound for the principal curvatures on $L_{t}$, i.e., $\kappa_{m}(p) \leq$ $\beta_{t}$ for any $p \in L_{t}$ (by conditions of Subsection 4.5, we can take $\beta(t)=\alpha / t$, but to simplify calculations we shall substitute this only at the very end).

(iii) $H(x)=\kappa_{1}(x)+\kappa_{2}(x)+\cdots+\kappa_{m}(x)$ is the mean curvature of $L_{t}$ at $x \in L_{t}$, and

$$
H^{ \pm}(x)=\max \{0, \pm H(x)\}
$$

are the positive and negative parts of $H(x)$.

Note that the signs of $\kappa_{i}$ are chosen so as to ensure the following formula:

$$
A^{\prime}(t)=\int_{L_{t}} H /|\nabla f| \text {. }
$$

(iv) $G(x)=2 \sum_{i<j} \kappa_{i} \kappa_{j}$ is the external term in the Gauss formula for the scalar curvature of $L_{t}$, i.e.,

$$
\mathrm{Sc}_{L}=2 \sum_{i<j}\left\langle R_{M}\left(e_{i}, e_{j}\right) e_{j}, e_{i}\right\rangle+G=\operatorname{Sc}_{M}-2 \operatorname{Ric}_{M}(u, u)+G
$$

for an orthonormal frame $\left\{e_{i}\right\}$ of the tangent space to $L$.

\section{Trivial inequalities.}

(i) Let $L \subset M$ be a hypersurface. By the Gauss formula, we have

$$
\mathrm{Sc}_{L}=\mathrm{Sc}_{M}-2 \operatorname{Ric}_{M}(u, u)+G \text {. }
$$

Therefore,

$$
\begin{aligned}
\mathrm{Sc}_{M}^{+} & \leq \mathrm{Sc}_{L}^{+}+m(m-1) K_{M}^{-}+2 \operatorname{Ric}_{M}(u, u)-G \\
& \leq \mathrm{Sc}_{L}^{+}+m^{2} K_{M}^{-}+2 \operatorname{Ric}_{M}(u, u)-G
\end{aligned}
$$

and

$$
G \leq \mathrm{Sc}_{L}^{+}+(m-1)(m-2) K_{M}^{-} \leq \mathrm{Sc}_{L}^{+}+m^{2} K_{M}^{-}
$$

(ii) Again by the Gauss formula, we have

$$
K_{L}^{-} \leq K_{M}^{-}+\left(H^{-}+m \beta\right) \beta
$$

(iii) Clearly, $H^{+}(x) \leq m \beta_{t}$ for any $x \in L_{t}$, whence

$$
\int_{L_{t}} H^{+} \leq m \beta_{t} A(t)
$$

Thus,

$$
\int_{L_{t}} H^{-} \leq \int_{L_{t}} H^{-} /|\nabla f|=\int_{L_{t}} H^{+} /|\nabla f|-\int_{L_{t}} H /|\nabla f| \leq m \alpha \beta_{t} A(t)-A^{\prime}(t) .
$$

Intermediate inequality. First, we prove that

$$
\int_{f^{-1}([a, b])} \operatorname{Sc}_{L}^{+} \leq \operatorname{const}(q, k, \ell)\left[1+\int_{f^{-1}([a, b])} K_{M}^{-}\right] .
$$

Indeed, since $|\nabla f| \geq 1 / \alpha$, we can write

$$
\int_{f^{-1}([a, b])} \mathrm{Sc}_{L}^{+} \leq \alpha \int_{a}^{b} d t \int_{L_{t}} \mathrm{Sc}_{L}^{+}
$$

(applying $\mathrm{A}_{k}$ for $L_{t}$ )

$$
\leq \mathcal{A}(q, k, \ell) \alpha \int_{a}^{b} d t\left(1+\int_{L_{t}} K_{L_{t}}^{-}\right)
$$


(applying the trivial inequalities above)

$$
\begin{aligned}
& \leq \mathcal{A}(q, k, \ell) \alpha \int_{a}^{b} d t\left(1+\int_{L_{t}}\left[K_{M}^{-}+\left(H^{-}+m \beta_{t}\right) \beta_{t}\right]\right) \\
& \leq \mathcal{A}(q, k, \ell) \alpha\left[(b-a)+\int_{f^{-1}([a, b])} K_{M}^{-}+m(1+\alpha) \int_{a}^{b} A(t) \beta_{t}^{2}-\int_{a}^{b} A^{\prime}(t) \beta_{t}\right] .
\end{aligned}
$$

Now, since $0<a<b \leq 1, \alpha=\alpha(q, k, \ell), \beta_{t}=\alpha / t$, and $A(t) \leq \alpha t^{m}$, we obtain $(\diamond)$.

\section{Main inequality.}

$$
\int_{f^{-1}([a, b])} \operatorname{Sc}_{M}^{+} \leq \int_{f^{-1}([a, b])} \operatorname{Sc}_{L}^{+}+m^{2} \int_{f^{-1}([a, b])} K_{M}^{-}+2 \int_{f^{-1}([a, b])} \operatorname{Ric}(u, u)-\int_{f^{-1}([a, b])} G
$$

(by the relative Bochner formula)

$$
\leq \int_{f^{-1}([a, b])} \operatorname{Sc}_{L}^{+}+m^{2} \int_{f^{-1}([a, b])} K_{M}^{-}+\int_{f^{-1}([a, b])} G+2 \int_{L_{a}} H-2 \int_{L_{b}} H
$$

(applying the trivial inequality (i))

$$
\leq 2 \int_{f^{-1}([a, b])} \operatorname{Sc}_{L}^{+}+2 m^{2} \int_{f^{-1}([a, b])} K_{M}^{-}+2 \int_{L_{a}} H^{+}+2 \int_{L_{b}} H^{-}
$$

(applying the intermediate inequality $(\diamond)$ and estimates for the integrals of $H^{ \pm}$)

$$
\begin{aligned}
& \leq \operatorname{const}(q, k, \ell)\left[1+\int_{f^{-1}([a, b])} K_{M}^{-}\right]+m \beta_{a} A(a)+m \alpha \beta_{b} A(b)-A^{\prime}(b) \\
& \leq \operatorname{const}(q, k, \ell)\left[1+\int_{f^{-1}([a, b])} K_{M}^{-}\right]-A^{\prime}(b) .
\end{aligned}
$$

In particular, for any $\tau \in\left[b, \frac{3}{2} b\right]$ we have

$$
\int_{f^{-1}([a, b])} \operatorname{Sc}_{M}^{+} \leq \operatorname{const}(q, k, \ell)\left[1+\int_{f^{-1}\left(\left[a, \frac{3}{2} b\right]\right)} K_{M}^{-}\right]-A^{\prime}(\tau) .
$$

Since $0 \leq A(t) \leq \alpha t^{m}$ (see the conditions after $(* *)$ ), we conclude that for some $\tau \in\left[b, \frac{3}{2} b\right]$ we have $\left(-A^{\prime}(\tau)\right) \leq 2 \alpha b^{m-1}$. Therefore, we obtain $(* *)$.

4.6. $\mathrm{B}_{k} \Rightarrow \mathrm{A}_{k}^{\prime}$. We argue by contradiction, assuming that $\mathrm{A}_{k}^{\prime}$ is false. Then there is a sequence of $q$-manifolds $N_{n}$ with sectional curvature at least -1 and with $\left(k, \delta_{k}, \ell\right)$-corner surfaces $M_{n} \subset N_{n}$ determined by strainers $\left\{f_{n, i}, g_{n, i}\right\}$, and a sequence of points $x_{n} \in N_{n}$ such that

$$
\frac{\int_{M_{n} \cap B_{1}\left(x_{n}\right)} \mathrm{Sc}_{M_{n}}^{+}}{1+\int_{M_{n} \cap B_{2}\left(x_{n}\right)} K_{M_{n}}^{-}} \rightarrow \infty \text { as } n \rightarrow \infty .
$$

Passing to a subsequence of $N_{n}$, we may assume the following convergences (see Subsection 3.2):

(i) $\left(N_{n}, x_{n}\right) \rightarrow(N, x)$, where $N$ is an Aleksandrov space of dimension at most $q$ and curvature at least -1 ;

(ii) $f_{i, n} \rightarrow f_{i}: N \rightarrow \mathbb{R}$ and $g_{i, n} \rightarrow g_{i}: N \rightarrow \mathbb{R}$ for each $i \in\{1, \ldots, k\}$.

(iii) $M_{n} \rightarrow M \subset N$, where $M$ is a $\left(k, \delta_{k}, \ell\right)$-corner surface with strainers $f_{i}, g_{i}$ : $N \rightarrow \mathbb{R}$. 
Case with no spires. Suppose that $M$ has no spires (see Subsection 3.5). Then, since the set $M \cap \bar{B}_{1}(x) \subset N$ is compact, it can be covered by a finite number of "annuli"

$$
\operatorname{Ann}_{x_{i}}=\left\{y \in M|0<| x_{i} y \mid<b\left(x_{i}\right)\right\}, i=\{1,2, \ldots, s\} .
$$

For each $i \in\{1,2, \ldots, s\}$, we choose a sequence $x_{i, n} \in M_{n}$ converging to $x_{i} \in M$. Set $b_{i}=b\left(x_{i}\right)$ and $a_{i, n}=a_{\delta_{k}, b_{i}}\left(x_{i, n}\right)$ (see Subsection 3.7). In accordance with 3.7, $a_{i, n} \rightarrow 0$ as $n \rightarrow \infty$. Then, if $n$ is large, applying $\mathrm{B}_{k}$ for each $x_{i, n}$ with the pair $\left(a_{i, n}, b_{i}\right)$, we get

$$
\begin{aligned}
& \int_{M_{n} \cap B_{1}\left(x_{n}\right)} \operatorname{Sc}_{M_{n}}^{+} \leq \sum_{i=1}^{s} \int_{\left\{y \in M_{n}\left|2 a_{i, n}<\right| x_{i, n} y \mid<b_{i}\right\}} \operatorname{Sc}_{M_{n}}^{+} \\
& \leq \sum_{i=1}^{s} \mathcal{B}_{k}\left[1+\int_{\left\{y \in M_{n}\left|a_{i, n}<\right| x_{i, n} y \mid<2 b_{i}\right\}} K_{M_{n}}^{-}\right] \leq \mathcal{B}_{k} s\left[1+\int_{M_{n} \cap B_{2}\left(x_{n}\right)} K_{M_{n}}^{-}\right] .
\end{aligned}
$$

Therefore, we get a contradiction with $(\star)$.

Case with spires. First, note that from $M \cap \bar{B}_{1}(x)$ we can remove a finite set of spires (see Subsection 3.4) such that the remaining part can be covered by finitely many annuli:

$$
\left(M \backslash\left\{x_{1}, x_{2}, \ldots, x_{s}\right\}\right) \cap \bar{B}_{1}(x) \subset \bigcup_{i=1}^{S} \operatorname{Ann}_{x_{i}}, \quad S \geq s .
$$

Here the centers $x_{i}$ for $i \leq s$ are all spires, and for $i>s$, they are not. Then applying the same estimate as before, we get

$$
\begin{array}{r}
\int_{M_{n} \cap B_{1}\left(x_{n}\right)} \operatorname{Sc}_{M_{n}}^{+}-\sum_{i=1}^{s} \int_{\left\{y \in M_{n}|| \tilde{x}_{i, n} y \mid<2 a_{i, n}\right\}} \operatorname{Sc}_{M_{n}}^{+} \leq \sum_{i=1}^{S} \int_{\left\{y \in M_{n}\left|2 a_{i, n}<\right| \tilde{x}_{i, n} y \mid<b_{i}\right\}} \operatorname{Sc}_{M_{n}}^{+} \\
\leq \sum_{i=1}^{S} \mathcal{B}_{k}\left[1+\int_{\left\{y \in M_{n}\left|a_{i, n}<\right| \tilde{x}_{i, n} y \mid<b_{i}\right\}} K_{M_{n}}^{-}\right] \leq \mathcal{B}_{k} S\left[1+\int_{M_{n} \cap B_{2}\left(x_{n}\right)} K_{M_{n}}^{-}\right] .
\end{array}
$$

Property $(\star)$ implies that we can find $i \leq s$ such that

$$
\frac{\int_{M_{n} \cap B_{2 a_{i, n}\left(\tilde{x}_{i, n}\right)}} \operatorname{Sc}_{M_{n}}^{+}}{1+\int_{M_{n} \cap B_{4 a_{i, n}\left(\tilde{x}_{i, n}\right)}} K_{M_{n}}^{-}} \rightarrow \infty .
$$

Clearly in this case $a_{i, n}>0$ for all large $n$, and $a_{i, n} \rightarrow 0$ as $n \rightarrow \infty$.

We fix such a number $i$ and pass to shorter notation:

$$
x:=x_{i}, b:=b_{i}, x_{n}:=x_{i, n}, a_{n}:=a_{i, n}, \text { and so on. }
$$

Consider the rescaling $N_{n}^{\prime}=\frac{1}{2 a_{n}} N_{n}$. We denote by $M_{n}^{\prime}$ the image of $M_{n}$ in $N_{n}^{\prime}$ and, abusing notation, keep the same symbol for objects in $N_{n}$ and $N_{n}^{\prime}$ that correspond to each other. Note that

$$
\begin{aligned}
& \int_{M_{n}^{\prime} \cap B_{1}\left(x_{n}\right)} \operatorname{Sc}_{M_{n}^{\prime}}^{+}=\frac{1}{\left(2 a_{n}\right)^{n-2}} \int_{M_{n} \cap B_{2 a_{n}}\left(x_{n}\right)} \operatorname{Sc}_{M_{n}}^{+}, \\
& \int_{M_{n}^{\prime} \cap B_{2}\left(x_{n}\right)} K_{M_{n}^{\prime}}^{-}=\frac{1}{\left(2 a_{n}\right)^{n-2}} \int_{M_{n} \cap B_{4 a_{n}}\left(x_{n}\right)} K_{M_{n}}^{-} .
\end{aligned}
$$

Therefore, since $a_{n} \rightarrow 0$, we have

$$
\frac{\int_{M_{n} \cap B_{2 a_{n}}\left(\tilde{x}_{n}\right)} \operatorname{Sc}_{M_{n}}^{+}}{1+\int_{M_{n} \cap B_{4 a_{n}}\left(\tilde{x}_{n}\right)} K_{M_{n}}^{-}} \rightarrow \infty \text { as } n \rightarrow \infty .
$$


This implies that

$$
\frac{\int_{M_{n}^{\prime} \cap B_{1}\left(\tilde{x}_{n}\right)} \operatorname{Sc}_{M_{n}^{\prime}}^{+}}{1+\int_{M_{n}^{\prime} \cap B_{2}\left(\tilde{x}_{n}\right)} K_{M_{n}^{\prime}}^{-}} \rightarrow \infty \text { as } n \rightarrow \infty .
$$

Passing to a subsequence if necessary, we can assume that $\left(N_{n}^{\prime}, x_{n}^{\prime}\right) \rightarrow\left(N^{\prime}, x^{\prime}\right)$ and repeat for $N^{\prime}$ the same procedure as for $N$. It only remains to check the following.

4.7. Claim. There may be only finitely many such repetitions. In other words, after a finite number of repetitions, we arrive at the case with no spires.

This claim follows from the fact that $\operatorname{dir}_{\delta_{k}} N^{\prime}>\operatorname{dir}_{\delta_{k}} N$ (see Property 3.6) and the fact that $\operatorname{dir}_{\delta_{k}} N$ is an integer and $\operatorname{dir}_{\delta_{k}} N \leq \operatorname{dir}_{\delta_{k}}\left(\mathbb{R}^{q}\right)<\infty$; see Subsection 3.3 .

\section{ACKNOWLEDGMENTS}

I would like to thank Nina Lebedeva and Yurii Burago for their comments on the original version of this paper and for indication of mistakes; I am also thankful to Fyodor Zarkhin for correcting my English.

\section{REFERENCES}

[BGP] Yu. D. Burago, M. L. Gromov, and G. Ya. Perel'man, A. D. Aleksandrov spaces with curvatures bounded below, Uspekhi Mat. Nauk 47 (1992), no. 2, 3-51; English transl., Russian Math. Surveys 47 (1992), no. 2, 1-58. MR.1185284 (93m:53035)

[Buy] S. V. Buyalo, Some analytic properties of convex sets in Riemannian spaces, Mat. Sb. (N.S.) 107 (149) (1978), no. 1, 37-55; English transl. in Math. USSR-Sb. 35 (1978). MR0510141 (80a:53071)

[P-2003] A. Petrunin, Polyhedral approximations of Riemannian manifolds, Turkish J. Math. 27 (2003), no. 1, 173-187. MR.1975337 (2004f:53035)

[P-2007] _ Semiconcave functions in Alexandrov's geometry, Surveys in Differential Geometry, vol. 11, Internat. Press, Somerville, MA, 2007, pp. 137-201. MR2408266

Department of Mathematics, Pennsylvania State University, University Park, PennsylvaNIA 16802

E-mail address: petrunin@math.psu.edu

Received 5/APR/2007

Translated by THE AUTHOR 